DRAFT VERSION NOVEMBER 6, 2020

Typeset using LATEX twocolumn style in AASTeX63

\title{
NEAR-INFRARED SPECTROSCOPY OF CK VULPECULAE: REVEALING A REMARKABLY POWERFUL BLAST FROM THE PAST
}

\author{
D. P. K. Banerjee, ${ }^{1}$ T. R. Geballe, ${ }^{2}$ A. Evans,${ }^{3}$ M. Shahbandeh,${ }^{4}$ C. E. Woodward,${ }^{5}$ R. D. Gehrz, 5 S. P. S. Eyres,${ }^{6}$ \\ S. STARRFIELD, ${ }^{7}$ AND A. ZiJlstra ${ }^{8}$ \\ ${ }^{1}$ Astronomy \& Astrophysics Division, Physical Research Laboratory, Navrangpura, Ahmedabad, 380009, India \\ ${ }^{2}$ Gemini Observatory/NSF's NOIRLab, 670 N. A'ohoku Place, Hilo, Hawai'i, 96720, USA \\ ${ }^{3}$ Astrophysics Group, Lennard Jones Laboratories, Keele University, Keele, Staffordshire, ST5 5BG, UK \\ ${ }^{4}$ Department of Physics, Florida State University, 77 Chieftain Way, Tallahassee, FL 32306-4350, USA \\ ${ }^{5}$ Minnesota Institute for Astrophysics, School of Physics \& Astronomy, 116 Church Street SE, University of Minnesota, Minneapolis, MN 55455, USA \\ ${ }^{6}$ Faculty of Computing, Engineering, and Science, University of South Wales, Pontypridd, CF37 1DL, UK \\ ${ }^{7}$ School of Earth and Space Exploration, Arizona State University, Box 871404, Tempe, AZ 85287-1404, USA \\ ${ }^{8}$ Jodrell Bank Centre for Astrophysics, School of Physics and Astronomy, University of Manchester, Manchester M13 9PL, UK
}

\begin{abstract}
CK Vulpeculae, which erupted in AD 1670-71, was long considered to be a nova outburst; however, recent observations have required that alternative scenarios be considered. Long slit infrared spectroscopy of a forbidden line of iron reported here has revealed high line-of-sight velocities $\left(\sim \pm 900 \mathrm{~km} \mathrm{~s}^{-1}\right)$ of the ansae at the tips of the bipolar lobes imaged in $\mathrm{H} \alpha$ in 2010. The deprojected velocities of the tips are approximately $\pm 2130 \mathrm{~km} \mathrm{~s}^{-1}$ assuming the previously derived inclination angle of $65^{\circ}$ for the axis of cylindrical symmetry of the bipolar nebula. Such high velocities are in stark contrast to previous reports of much lower expansion velocities in CK Vul. Based on the deprojected velocities of the tips and their angular expansion measured over a 10-year baseline, we derive a revised estimate, with estimated uncertainties, of $3.2_{-0.6}^{+0.9} \mathrm{kpc}$ for the distance to CK Vul. This implies that the absolute visual magnitude at the peak of the 1670 explosion was $M_{V}=-12.4_{-2.4}^{+1.3}$, indicating that the 1670 event was far more luminous than previous estimates and brighter than any classical nova or any Galactic stellar merger. We propose that CK Vul belongs to the class of Intermediate Luminosity Optical Transients (ILOTs), objects which bridge the luminosity gap between novae and supernovae. While eruptions in lower luminosity ILOTs are attributed to merger events, the origin of the highly luminous ILOT outbursts is currently not known.
\end{abstract}

Keywords: stars: individual (CK Vul) — stars: peculiar — stars: winds, outflows —infrared: stars

\section{INTRODUCTION}

CK Vulpeculae (long referred to as Nova Vulpeculae 1670) was believed until recently to possibly be the earliest documented nova. It was discovered as a naked-eye object on 1670 June 20 by Anthelme, and independently on July 25 by Hevelius. Reconstruction of its light curve (Shara et al. 1985) shows it had a visual brightness maximum of approximately 3 mag at discovery, followed successively by a fading and a second maximum at approximately $2.6 \mathrm{mag}$ in 1671 March. It subsequently faded from naked-eye view, although a final maximum of 5.5-6 mag was observed by Hevelius in 1672 March. Since 1679, attempts to recover the object were unsuccessful until the discovery in 1982 of a small patch of $\mathrm{H} \alpha$ nebulosity at the site of the 1670 event (Shara \& Moffat 1982). A thorough discussion is given by Shara et al. (1985).

dpkb12345@gmail.com
Submillimeter photometry of CK Vul (Evans et al. 2002) revealed a large excess due to emission from dust, inconsistent with CK Vul being a classical nova remnant. A faint $70^{\prime \prime}$ bipolar nebula, oriented almost exactly N-S (Figure 1), was discovered in $\mathrm{H} \alpha$ imaging by Hajduk et al. (2007), who also reported a compact radio source located at the point of symmetry. Infrared (IR) spectroscopy (Evans et al. 2016) revealed a rich spectrum of hydrocarbons, together with $\mathrm{H}_{2}$, $\mathrm{HCN}$ and metallic fine structure line emission. $\mathrm{A} \sim 10^{\prime \prime}$ dusty bipolar submillimeter structure, symmetric about a small disk-like feature, the center of which coincides with the compact radio source, was reported by Eyres et al. (2018). This structure, which aligns with dark lanes in the $\mathrm{H} \alpha$ image, also shows emission from several small organic molecules.

The central star of CK Vul is deeply enshrouded in dust, and is visible in neither the optical nor the near-IR. The current identification of CK Vul in the SIMBAD database is incorrect. In this study, we adopt the radio coordinates (Hajduk et al. 2007) listed in Table 1 for the central source of CK Vul. 


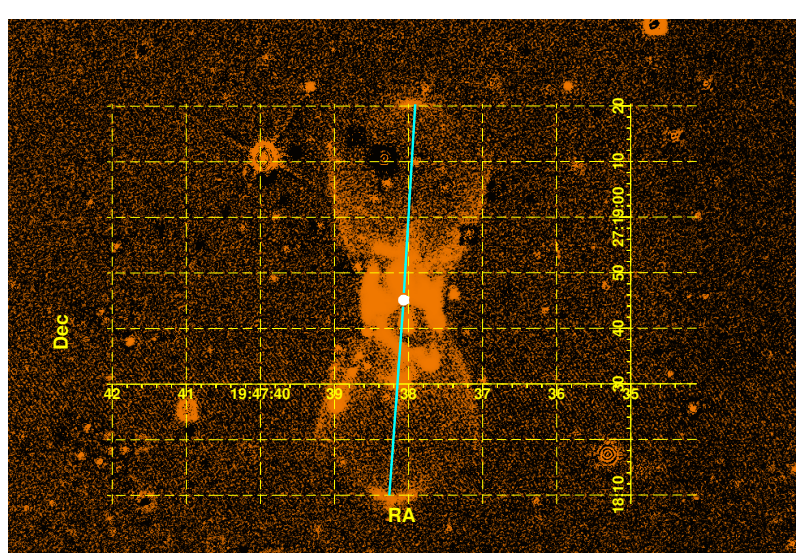

Figure 1. $\mathrm{H} \alpha$ continuum-subtracted image of CK Vul observed on 2010 June 22 with the Gemini North Telescope (image kindly provided by M. Hajduk) . The position of the compact radio source is indicated by the white dot. The slit (cyan line) was centered on this source and passes through the tips of the ansae located near the top and bottom center of the image.

No consensus exists on the cause of the 1670 eruption. Proposed scenarios include a nova eruption, various types of stellar merger, and a very late thermal pulse in a post-AGB star (Shara \& Moffat 1982; Shara et al. 1985; Hajduk et al. 2007; Kamiński et al. 2015, 2017, 2018a; Eyres et al. 2018). A kinematic study of the nebula could be a means of discriminating between some of the above mechansims. For example, stellar mergers rarely produce outflows with velocities exceeding $500 \mathrm{~km} \mathrm{~s}^{-1}$. On the other hand, nova eruptions can generate ejecta velocities of up to several thousand $\mathrm{km} \mathrm{s}^{-1}$.

Here we present near-IR spectra of CK Vul, obtained through a slit positioned as shown in Figure 1. The orientation was chosen so that the slit intercepted the radio source at the center of the bipolar structure and the northern and southern tips of the ansae, approximately $36^{\prime \prime}$ from the center, where the expansion velocities are expected to be largest in a homologous or Hubble flow (Balick \& Frank 2002, and references therein).

\section{OBSERVATIONS AND DATA REDUCTION}

Spectroscopy of CK Vul was obtained at the Frederick C. Gillett Gemini North Telescope on Maunakea on the nights of 202031 August and 1 September (UT), using the Gemini Near-InfraRed Spectrograph (GNIRS; Elias et al. 2006). Sky conditions were excellent throughout the observations. The spectrograph configuration included its $99^{\prime \prime}$ slit oriented $3^{\circ}$ west of north, a slit width of $0.45^{\prime \prime}$, and the short focal length blue camera, which provides a plate scale of $0.15^{\prime \prime} /$ array pixel. The $110.5 \mathrm{\ell} / \mathrm{mm}$ grating in GNIRS was set to a central wavelength of $1.26 \mu \mathrm{m}$, which gave a coverage of $1.203-1.317 \mu \mathrm{m}$ and a resolving power, $R$, of 4800 (corresponding to resolutions of $0.00026 \mu \mathrm{m}$ and $62 \mathrm{~km} \mathrm{~s}^{-1}$ ).

Individual exposure times were 300 seconds. The total exposure times on source were 1800 seconds on 31 August and 2400 seconds on 1 September. The same exposure times were used at a location $46^{\prime \prime}$ to the east, selected to minimize the number of stars in the "sky" slit. Exposures were obtained in a the standard ABBA manner. The star HIP 98699 (A1V) was observed immediately following CK Vul; the airmass match was within 0.04 airmasses on each night.

On both nights the slit was centered on the radio coordinates (Table 1 ) using a blind offset from a $K \approx 10$ mag star located $0.6^{\prime}$ distant. The offsetting accuracy of Gemini is considerably less than a GNIRS pixel. Although CK Vul itself was not detected in the near-IR, we are confident of the angular distances from it to the spectral features reported in this paper.

Examination of raw sky-subtracted frames revealed within $10^{\prime \prime}$ of CK Vul several patches of emission along the slit in the forbidden line of Fe II at $1.25702 \mu \mathrm{m}$ (vacuum). The H I $\mathrm{Pa} \beta$ line at $1.28216 \mu \mathrm{m}$ was also weakly present in a portion of this region within a few arcseconds of the radio source.

Prompted by the report by Hajduk et al. (2013) of $\mathrm{H} \alpha$ line emission with high proper motions at the northern and southern tips of the bipolar nebula, we searched the spectral images on both nights and found regions of faint and highly Doppler-shifted $\left(\sim \pm 900 \mathrm{~km} \mathrm{~s}^{-1}\right)$ emission in the [Fe II] line, about one arcsecond in extent, close to the locations of the $\mathrm{H} \alpha$ tips. The northern emission stands out fairly well. The southern emission has superimposed on part of it the spectrum of a faint star. However, careful data reduction clearly established the emission's existence. We have considered whether these components are lines of some other atomic species, but think it highly unlikely and unreasonable that an atomic species would selectively create only one-sided emission (i.e. emission in only one of the two ansae) and also that emission would appear only in the tips and not in the bright central region where it would be expected to be strong. Thus, we are confident in our identification.

Full reduction of the spectra from each night was limited to the wavelength interval containing the $[\mathrm{Fe}$ II] and $\mathrm{Pa} \beta$ lines, there being no detected lines outside of that interval. It involved the standard steps of flat-fielding, spatial and spectral rectification of the images, removal of the effects of cosmic ray hits, wavelength calibration using an argon lamp, and binning of the spectra, in this case into intervals of $0.0002 \mu \mathrm{m}$ $\left(48 \mathrm{~km} \mathrm{~s}^{-1}\right)$.

Spectra of the twelve regions where the [Fe II] line was detected were extracted from the rectified CK Vul reduced image from each night. Each was flux-calibrated by dividing by the spectrum of the standard star, including correcting for slit loss in the spectrum of the star. The spectra from the two nights were then combined to produce a final spectrum for each region. Pertinent information for each spectrum is provided in Table 1. 
Table 1. Observed [Fe II] $1.257 \mu \mathrm{m}$ line.

\begin{tabular}{lcccc}
\hline \hline Offset $^{a}$ & $\begin{array}{c}\text { Aperture } \\
\text { arcsec }\end{array}$ & $\begin{array}{c}\text { Vel. of peak }^{b} \\
\text { arcsec }\end{array}$ & $\begin{array}{c}\text { Flux }^{c} \\
\mathrm{~km} \mathrm{~s}^{-1} \text { hel. }\end{array}$ & $\begin{array}{c}\text { Notes } \\
10^{-19} \mathrm{~W} \mathrm{~m}^{-2}\end{array}$ \\
\hline+36.7 & $0.45 \times 0.90$ & +869 & 1.39 & blue shoulder \\
+7.9 & $0.45 \times 0.75$ & -111 & 0.53 & \\
+4.2 & $0.45 \times 0.75$ & -147 & 1.48 & blue shoulder \\
+4.2 & $0.45 \times 0.75$ & -40 & 2.15 & \\
+2.9 & $0.45 \times 0.75$ & -118 & 6.25 & \\
+1.5 & $0.45 \times 0.75$ & -106 & 5.53 & $\mathrm{~Pa} \beta$ at $-120 \mathrm{~km} \mathrm{~s}^{-1}$ \\
+0.6 & $0.45 \times 0.45$ & -300 & 0.84 & \\
+0.6 & $0.45 \times 0.45$ & -63 & 2.62 & $\mathrm{~Pa} \beta$ at $-50 \mathrm{~km} \mathrm{~s}^{-1}$ \\
0.0 & $0.45 \times 0.45$ & -302 & 1.16 & \\
0.0 & $0.45 \times 0.45$ & -47 & 0.56 & Pa $\beta$ at $-50 \mathrm{~km} \mathrm{~s}^{-1}$ \\
-0.6 & $0.45 \times 0.45$ & -285 & 0.25 & \\
-3.3 & $0.45 \times 0.75$ & +68 & 2.25 & \\
-4.1 & $0.45 \times 0.75$ & +20 & 3.50 & \\
-8.9 & $0.45 \times 0.75$ & +137 & 2.05 & \\
-37.1 & $0.45 \times 1.20$ & -922 & 0.84 & may have red shoulder \\
\hline \hline
\end{tabular}

${ }^{a}$ From radio coordinates of CK Vul $(J 2000 \mathrm{RA}=19: 47: 38.074$, Dec $=+27: 18: 45.16)$ along slit at position angle 3 deg west of north.

${ }^{b}$ Uncertainty $\sim 10 \mathrm{~km} \mathrm{~s}^{-1}$.

${ }^{c}$ Typical random uncertainty $0.10 \mathrm{~W} \mathrm{~m}^{-2}$. Uncertainty in absolute flux calibration $\sim 20$ percent.

\section{RESULTS}

Spectra of [Fe II] $1.257 \mu \mathrm{m}$ at all positions where the line was detected are presented in Figure 2. Emission was also detected in the $\mathrm{Pa} \beta$ line, but only close to the radio source. A third line, [Fe II] at $1.279 \mu \mathrm{m}$, was also weakly detected, but only in a few arcsecond long segment of the slit where the $1.257 \mu \mathrm{m}$ line is at its brightest. Figure 3 shows the spectrum of that portion of the central region where all three lines are present.

As can be seen in Figure 2, the [Fe II] $1.257 \mu$ m line emission is prominent at various locations within $\pm 9^{\prime \prime}$ of the central source. The velocity pattern within this inner region is complex, with large variations with position and at some locations more than one component present. Discussion and modeling of the velocity field will be reported elsewhere.

The highlight of these observations is the high line-ofsight velocities observed in the tips of the north and south ansae in the [Fe II] line, peaking at $\sim+870 \mathrm{~km} \mathrm{~s}^{-1}$ and $\sim$ $-920 \mathrm{~km} \mathrm{~s}^{-1}$, respectively. Hajduk et al. (2013), using the morpho-kinematic modelling tool SHAPE (Steffen \& López 2006) found that that inclination angle of the bipolar $\mathrm{H} \alpha$ nebula is $65^{\circ}$ relative to the line-of-sight. That implies that the northern and southern tips are moving outward from the center at $2130 \mathrm{~km} \mathrm{~s}^{-1}\left(900 \mathrm{~km} \mathrm{~s}^{-1} / \cos 65^{\circ}\right)$. This is vastly higher than previously reported expansion velocities in CK Vul, which are of order a few hundred $\mathrm{km} \mathrm{s}^{-1}$ or less (Shara et al. 1985; Hajduk et al. 2007).

The reason for these previously reported low velocities is now clear: all of them are for regions located around the central waist of the hourglass nebula (i.e. the equatorial region) where low expansion velocities are expected. Hourglass or bipolar nebulae are believed to be generated when the outflowing ejecta from the central source are constricted by a material over-densities in the equatorial planes, i.e. at the waist of the hourglasses. The ejecta expand freely and rapidly in the polar directions, but slowly in the equatorial region. High velocities are expected in the polar regions by virtue of the larger distance to the tips of the ansae.

For the $\mathrm{H} \alpha$ frame of 2010 June 22 (Figure 1) we measure the angular separation between the centers of the tips of the ansae at the positions where the GNIRS slit inter-

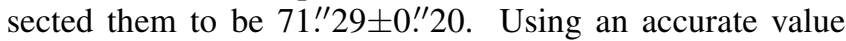
of $0 .{ }^{\prime \prime} 1517 \pm 0$. $^{\prime} 0002$ pixel $^{-1}$ for the plate scale of GNIRS in the configuration in which it was used, we find an angular separation of 73 !' $87 \pm 0$. .' 23 for the centers of the tips in the [Fe II] $1.257 \mu \mathrm{m}$ emission line. Because [Fe II] emission arises in a region where hydrogen is partially ionized (Mouri et al. 2000), we assume that $\mathrm{H} \alpha$ and [Fe II] are cospatial. An expansion of 2 ." $58 \pm 0$.' 30 has therefore occurred over a 10.2 year period (2010 June 22 to 2020 August 31). Assuming constant velocity this implies that the expansion began $290 \pm 40$ years ago. Comparison with the the likely date of the ejection event, 1670 or 1671 , suggests that the deceleration of the tips, if any, has been rather modest.

Assuming a constant transverse velocity of $1930 \mathrm{~km} \mathrm{~s}^{-1}$ $\left(2130 \sin 65^{\circ}\right.$ ) between 2010 and 2020 (i.e., no significant deceleration of the ejecta), the distance to $\mathrm{CK}$ Vul is $3.2 \mathrm{kpc}$, independent of reddening. This distance is a factor 


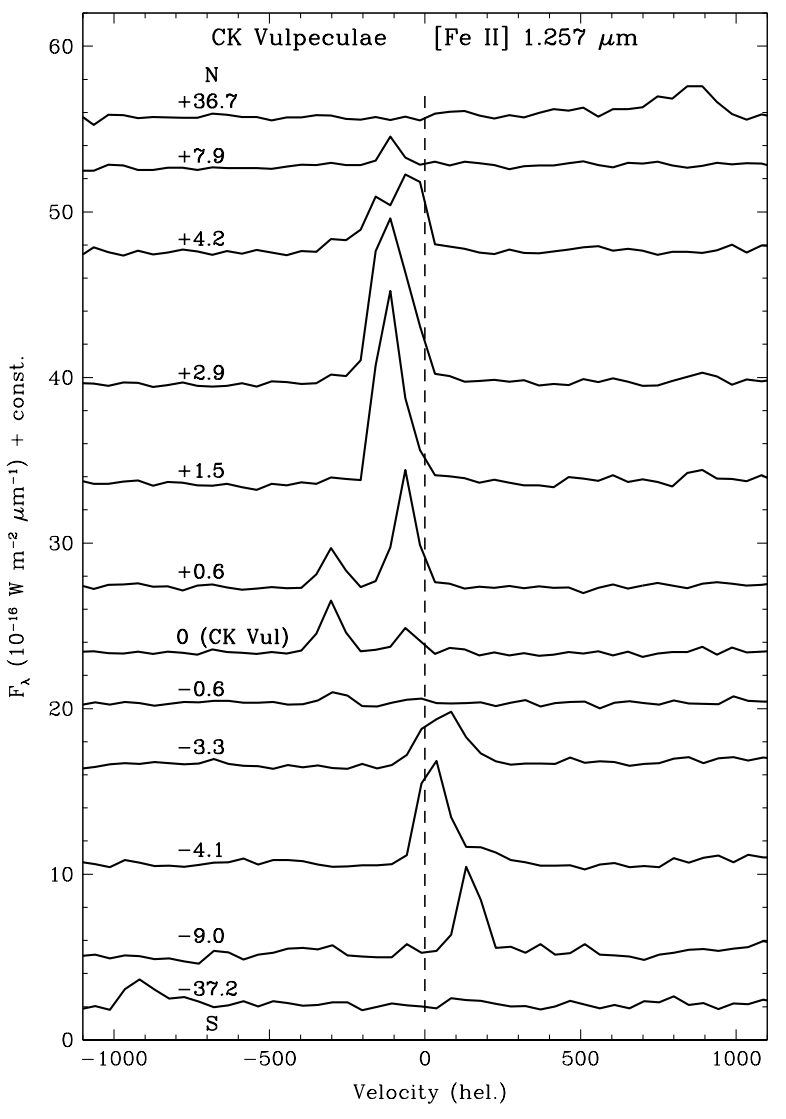

Figure 2. Spectra of the [Fe II] $1.257 \mu \mathrm{m}$ line in the CK Vul nebula at the twelve locations along the slit where the line was detected. See Table 1 for details.

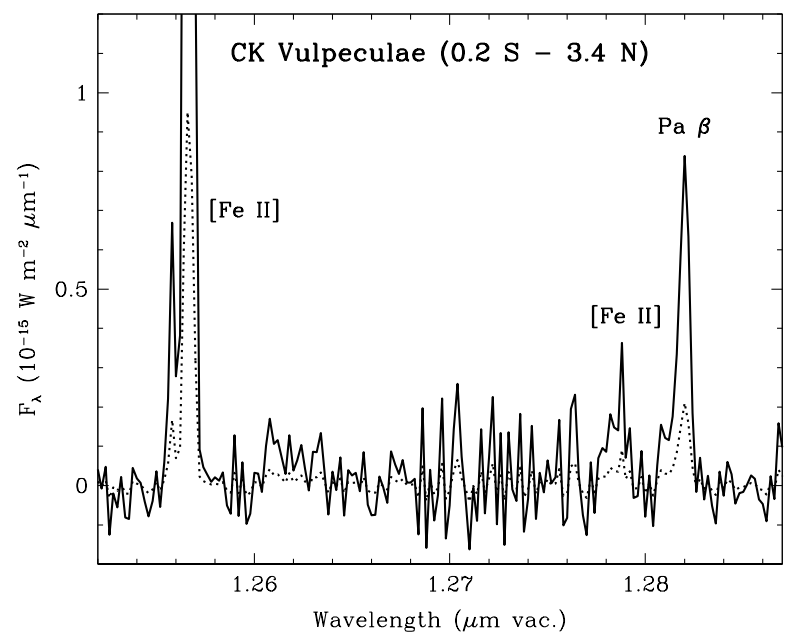

Figure 3. Spectrum extracted over a 3.' 6 region near the center, showing three lines ([Fe II] $1.257 \mu \mathrm{m}$ and $1.279 \mu \mathrm{m}$, and $\mathrm{HI} \mathrm{Pa} \beta$ $1.282 \mu \mathrm{m})$. Dotted line: same spectrum scaled down by a factor of four. of 4.5-6 times larger than other estimates (Shara et al. 1985; Hajduk et al. 2013).

The uncertainty in the derived inclination angle was not given by Hajduk et al. (2013) and is not incorporated in the above distance uncertainty. At high inclination angles, expansion velocity, distance, and absolute magnitude all have non-linear dependences on this angle. An uncertainty of $5^{\circ}$ in the assumed $65^{\circ}$ gives the distance to CK Vul as $3.2_{-0.6}^{+0.9} \mathrm{kpc}$; further allowing for the uncertainty in reddening gives the absolute visual magnitude at outburst as $M_{V}=-12.4_{-2.4}^{+1.3}$.

The above distance of $3.2_{-0.6}^{+0.9} \mathrm{kpc}$ is in apparent conflict with distance estimates by Hajduk et al. (2013) of $\sim 500 \mathrm{pc}$ and $\sim 2 \mathrm{kpc}$ to two variable field stars, which they argued lie behind some of the ejecta of CK Vul, which has caused their recent brightness fluctuations and apparent Li overabundances. However, the former of these has a Gaia parallax (Bailer-Jones et al. 2018) of $4.9 \mathrm{kpc}$ and thus no longer conflicts. The latter star is too faint for Gaia and a more precise distance would need to be determined by some other means.

\section{DISCUSSION}

\subsection{Line emission in the central few arcseconds}

Here we confine our discussion to the central region and the spectrum in Figure 3, in which all three lines ([Fe II] 1.257 and $1.279 \mu \mathrm{m}$, and $\mathrm{Pa} \beta$ ) are present. This spectrum includes contributions from several disparate areas. Thus the properties derived here are mean values and do not necesaarly represent any individual region. Based on the strength of [Fe II] $1.257 \mu \mathrm{m}$ relative to $\mathrm{Pa} \beta$, we assume that the gas in this region is largely collisionally excited. However, the presence of a compact radio source (Hajduk et al. 2007) indicates the possibility of some photoionization.

As discussed by Mouri et al. (2000), the [Fe II] emission arises in a region in which hydrogen is partially ionized. The critical density for collisional de-excitation of the upper level of the $1.257 \mu \mathrm{m}$ line is

$$
n_{\text {crit }}=5.6 \times 10^{4}\left(\frac{T_{e}}{10,000 \mathrm{~K}}\right)^{0.66} \mathrm{~cm}^{-3},
$$

where $T_{e}$ is the electron temperature. Useful diagnostics are the [Fe II] $1.257 \mu \mathrm{m} / \mathrm{Pa} \beta$ and [Fe II] $1.279 \mu \mathrm{m} /[\mathrm{Fe}$ II] $1.257 \mu \mathrm{m}$ flux ratios, which are $4.9 \pm 0.6$ and $0.07 \pm 0.02$ respectively.

The [Fe II] $1.257 \mu \mathrm{m} / \mathrm{Pa} \beta$ flux ratio suggests shock velocities of $\sim 70 \pm 5 \mathrm{~km} \mathrm{~s}^{-1}$ marginally below the threshold $\left(75 \mathrm{~km} \mathrm{~s}^{-1}\right)$ for an ionizing shock (Mouri et al. 2000). Note that this value is for solar abundances, which may not be applicable to these ejecta. The [Fe II] $1.279 \mu \mathrm{m} /[\mathrm{Fe} \mathrm{II}] 1.257 \mu \mathrm{m}$ flux ratio indicates $T_{e} \simeq$ $6000 \mathrm{~K}$, although the deduced $T_{e}$ is virtually independent of the flux ratio. This temperature gives a critical density of $n_{\text {crit }} \simeq 4 \times 10^{4} \mathrm{~cm}^{-3}$ for the upper level of the $1.257 \mu \mathrm{m}$ line.

\subsection{The outer tips}

The morphologies of the [Fe II] $1.257 \mu \mathrm{m}$ tips in the CK Vul nebula closely resemble the "fingertips" observed in 
the Orion Molecular Cloud by Bally et al. (2015, see e.g., their Fig. 9) in the shock-excited forbidden [Fe II] $1.644 \mu \mathrm{m}$ line. There over one-hundred high density clumps of gas are being ejected from the cloud at typical speeds of $300 \mathrm{~km} \mathrm{~s}^{-1}$, which Bally et al. concluded is the result of a violent event $\sim 500 \mathrm{yr}$ ago during the formation of a dense cluster of massive stars.

We propose that excitation of the upper level of the [Fe II] $1.257 \mu \mathrm{m}$ line in the CK Vul tips is the result of the same phenomenon as that proposed by Bally et al. (2015) for the [Fe II] line in Orion. In each of the tips a low velocity reverse shock is being driven into a high density gas clump ejected from CK Vul as each clump encounters much lower density interstellar gas. The shock ionizes and collisionally excites the Fe and, at least to some extent, the hydrogen. It is possible that lines of molecular hydrogen are also associated with these clunps, as has been observed in detail in Orion by Tedds et al. (1999). Observations of them could provide constraints on the physical properties of the clumps.

\subsection{The nature of $\mathrm{CK}$ Vul}

The greatly increased distance to CK Vul derived here has dramatic implications for the dust mass and luminosity in the bipolar nebula and for the luminosities of the explosive events in 1670-71. First, the dust mass in the compact bipolar dusty nebula reported by Eyres et al. (2018) now becomes $4.3 \times 10^{-3} \mathrm{M}_{\odot}$, of which $3.3 \times 10^{-3} \mathrm{M}_{\odot}$ lies in the diffuse emission, and $1.0 \times 10^{-3} \mathrm{M}_{\odot}$ lies in the disk. The present luminosity of the central object, as determined from the spectral energy distribution of the dust is $20 \mathrm{~L}_{\odot}$ (see Kamiński et al. 2015).

Second, assuming the maximum visual brightness $m_{V}$ in 1671 was $2.6 \pm 0.3$ (Shara et al. 1985), and that the visual extinction $A_{V}$ in the direction of CK Vul at a distance $d=$ $3.2 \mathrm{kpc}$ is $2.47 \pm 0.45$ (Marshall et al. 2006), the absolute magnitude of CK Vul at maximum was $-12.4_{-2.4}^{+1.3}$. It is thus clear that $C K$ Vul underwent a much more luminous sequence of explosions in 1670-71 than hitherto recognized. At peak its absolute magnitude was at least 3 mag brighter than the brightest Galactic classical nova, which generally have $M_{V}$ at maximum in the range -6.5 to -9.5 (see e.g. Bode \& Evans 2012). A few luminous extragalactic novae have been known to approach $M_{V}=-10$ (e.g. Kasliwal et al. 2011b).

CK Vul might be considered as an extreme example of a "Luminous Red Nova" (LRN; Kulkarni et al. 2007). Thus, it is of interest to compare its peak nebular expansion velocity of $\sim 2000 \mathrm{~km} \mathrm{~s}^{-1}$ with those of others of that class, such as V1309 Sco, V4332 Sgr and V838 Mon. V4332 Sgr produced an $\mathrm{H} \alpha$ line with full width at zero intensity $\sim$ $550 \mathrm{~km} \mathrm{~s}^{-1}$ (Martini et al. 1999), a far lower expansion velocity than CK Vul. Similarly, the P Cygni profiles in an early spectrum of V838 Mon (Lynch et al. 2004) have separations between their absorption and emission peaks of $540 \pm$ $140 \mathrm{~km} \mathrm{~s}^{-1}$. Recent millimeter/submillimeter-wave observations of V4332 Sgr, V838 Mon, and V1309 Sco taken 22, 14, and $8 \mathrm{yr}$, respectively, after their eruptions (Kamiński et al. 2018b), reveal molecular emission lines having full widths not exceeding $400 \mathrm{~km} \mathrm{~s}^{-1}$. In the few published spectra of extragalactic LRNe (e.g M31 RV and PTF10fqs) also only narrow emission lines are present, indicating velocities similar to those of their Galactic counterparts (Rich et al. 1989; Kasliwal 2012). In the case of PTF10fqs, in addition to the narrow Balmer and calcium emission features, there is a $10,000 \mathrm{~km} \mathrm{~s}^{-1}$ wide unidentified emission feature at $\sim 8600 \AA$, possibly suggestive of a terminal explosive event (Kasliwal et al. 2011a; Kasliwal 2012). In general, though, the highest velocities in CK Vul are considerably higher than those in LRNe.

The peak absolute luminosity of $\mathrm{CK}$ Vul is also much higher than the Galactic LRNe, the brightest of which was V838 Mon with $M_{V}=-9.8 \mathrm{mag}$ (Bond et al. 2003). Kasliwal (2012) has shown that there exists a group of objects with luminosities that are intermediate between the brightest novae and faintest supernovae (i.e. with peak $M_{V}$ between 9 and -14, Kasliwal 2012, Fig. 1). Soker \& Kashi (2011) have termed these Intermediate-Luminosity Optical Transients (ILOTs). In the literature ILOTs are interchangeably called Intermediate-Luminosity Red Transients or LRNe. V838 Mon, M31 RV, and M85OT are all proposed members of this group. It is suggested (Kasliwal 2012) that the less luminous of these outbursts result from mergers of main sequence stars: "mergeburst" events (Soker \& Tylenda 2007).

The strongest evidence for a mergeburst is the case of V1309 Sco, where a decaying orbital period prior to eruption was clearly detected (Tylenda et al. 2011), indicating the components of the binary were spiraling into each other. However, V1309 Sco is estimated to have a peak $M_{V}$ of only $\sim-6$ mag (although the distance to the object is uncertain).

To explain the outbursts of the more luminous ILOTs, alternative routes are needed. One suggested channel is electron capture in an $\mathrm{O}-\mathrm{Ne}-\mathrm{Mg}$ core in extreme asymptotic giant branch (eAGB) stars (Kasliwal 2012). The eAGB stars are massive AGB stars (5-10 $\mathbf{M}_{\odot}$, Soker \& Kashi 2011). Alternatively, Soker \& Kashi discuss the possibility that such outbursts could be related to major instabilities of the envelopes of eAGB stars leading to ejections of their envelopes. The focus on eAGB stars arises because the mid-IR progenitors of two of the nearest extragalactic ILOTs, NGC300OT and SN 2008S, are at the extremely luminous and red ends of the AGB branch (Prieto 2008; Thompson et al. 2009; Kasliwal 2012). Other suggested mechanisms are luminous blue variable eruptions, asteroids falling onto white dwarfs, accretion-induced collapses, peculiar core-collapse supernovae, and peculiar classical novae (Kasliwal 2012, and references therein).

We make no attempt to classify the nature of the CK Vul outburst (e.g., non-terminal Luminous Blue Variable, terminal events such as SN 2008S and NGC 300 OT2008-1). Discriminating between these is challenging even for present day events, despite having diagnostic tools such as panchromatic outburst spectra, archival images to identify the nature of the progenitor (e.g., Smith et al. 2010; Adams et al. 2016; Jencson et al. 2019; Andrews et al. 2020), and in some cases the presence of a stellar remnant. 
Any proposed mechanism/model to account for the properties of the CK Vul explosion must be able to account for its key features. These include the high peak outburst magnitude; the multiple peaks of the outburst; the sustained brightness of the outburst for a remarkably long period ( $\sim 700$ days; see the reconstructed light curve in Shara et al. 1985); the high peak expansion velocities exceeding $2000 \mathrm{~km} \mathrm{~s}^{-1}$; the formation and maintenance of jets, bubbles, and dust cocoons; and the production of the shortlived radio-nuclide ${ }^{26} \mathrm{Al}$ during its outburst (Kamiński et al. 2018a).

\section{ACKNOWLEDGMENTS}

This Letter is based on observations obtained for program GN-2020B-Q-207 at the international Gemini Observatory, a program of NSF's NOIRLab, which is managed by the Association of Universities for Research in Astronomy (AURA) under a cooperative agreement with the National Science Foundation on behalf of the Gemini Observatory partnership: the National Science Foundation (United States), National Research Council (Canada), Agencia Nacional de Investigación y Desarrollo (Chile), Ministerio de Ciencia, Tecnología e Innovación (Argentina), Ministério da Ciência, Tecnologia, Inovações e Comunicações (Brazil), and Korea Astronomy and Space Science Institute (Republic of Korea). DPKB is supported by a CSIR Emeritus Scientist grant-inaid, which is being hosted by the Physical Research Laboratory, Ahmedabad. We thank M. Hajduk for helpful comments on the manuscript.

Facilities: Gemini:Gillett

Software: Figaro (Shortridge et al. 1992), Gemini IRAF Package

\section{REFERENCES}

Adams, S. M., et al., 2016, MNRAS, 460, 1645

Andrews, J. E., et al., 2020, submitted to ApJ

(arXiv:2009.13541v1)

Bailer-Jones, C. A. L., Rybizki, J., Fouesneau, M., Mantelet, G., \& Andrae, R. 2018, AJ, 156, 58

Balick, B., \& Frank, A. 2002, ARA\&A, 40, 439

Bally, J., Ginsburg, A., Silvia, D., Youngblood, A. 2015, A\&A, 579, A130

Bode, M. F., Evans, A. 2012, eds Classical Novae, second edition, Cambridge University Press, Cambridge

Bond, H. E.,Henden, A., Levay, C. G., et al. 2003, Nature, 422, 405

Elias, J. H., Joyce, R. R., Liang, M., et al. 2006, in Ground-based and Airborne Instrumentation for Astronomy, eds I. S. McLean, M. Iye, Proceedings of the SPIE, 6269, 62694C

Evans, A., van Loon, J. Th., Zijlstra, A. A., et al. 2002 MNRAS, 332, L35

Evans, A., Gehrz, R. D., Woodward, C. E., et al. 2016 MNRAS, 457,2871

Eyres, S. P. S., Evans, A., Zijlstra, A., et al. 2018 MNRAS, 481, 4931

Hajduk, M., Zijlstra, A. A., van Hoof, P. A. M., et al. 2007, MNRAS, 378, 1298

Hajduk, M., van Hoof, P. A. M., \& Zijlstra, A. A. 2013 MNRAS, 432

Jencson, J. E., et al., 2019, ApJ, 880, L20

Kamiński, T., Menten, K. M., Tylenda, R., et al. 2015 Nature, 520, 322

Kamiński, T., Menten, K. M., Tylenda, R., et al. 2017 A\&A, 607, A78

Kamiński, T., Tylenda, R., Menten, K. M.,et al. 2018a, Nature Astronomy, 2, 778
Kamiński, T., Steffen, W., Tylenda, R., et al. 2018b, A\&A, 617, A129

Kasliwal, M., et al., 2011a, ApJ, 730, 134

Kasliwal, M. M., Cenko, S. B., Kulkarni, S. R., et al. 2011b, ApJ, 735, 94

Kasliwal, M. M. 2012, PASA, 29, 482

Kulkarni, S. R.,Ofek, E.O, Rau, A., et al. 2007, Nature, 447, 458

Lynch, D. K., Rudy, R. K., Russell, R. W., et al. 2004 ApJ, 607, 460

Marshall, D. J., Robin, A. C., Reylé, C., Schultheis, M., \& Picaud, S. 2006, A\&A, 453, 635

Martini, P., Wagner, R. M., Tomaney, A., Rich, R. M., della Valle, M., et al. 1999, AJ, 118, 1034

Mouri, H., Kawara, K., \& Taniguchi, Y. 2000,ApJ, 528, 186

Prieto, J. L. 2008, ATel \#1550

Rich, R. M., Mould, J., Picard, A., Frogel, J. A., \& Davies, R. 1989, ApJ, 341, L51

Shara, M., \& Moffat, A. F. J. 1982, ApJ, 258, L41

Shara, M., Moffat, A. F. J., \& Webbink, R. F. 1985, ApJ, 294, 271

Shortridge, K., Meyerdiercks, H., Currie, M., et al. 2004, Starlink Project, Starlink User Note 86.21

Smith, N., et al., 2010, AJ, 139, 1451

Soker, N. \& Tylenda, R. 2007, in The Nature of V838 Mon and its Light Echo, eds. R. L. M. Conrad \& U. Munari, ASP Conf Ser., 363,280

Soker, N., \& Kashi, A. 2011, arxiv 1107.3454

Steffen, W., \& López, J. A. 2006, RMxAA, 42, 99

Skrutskie, M. F., Cutri, R. M., Stiening, R., et al. 2006, AJ, 131, 1163

Tedds, J. A., Brand, P. W. J. L., \& Burton, M.G. 1999, MNRAS, 307,337 
Thompson, T. A., Prieto, J. L., Stanek, K. Z., Kistler, M. D., \& Beacom, J. F. 2009, ApJ, 705, 1364
Tylenda, R., Hajduk, M., \& Kamiński, T. 2011, A\&A, 528, A114 